\title{
Medication discrepancies among elderly patients discharged from a tertiary hospital: prevalence and risk factors
}

Izzati Abdul Halim Zaki ${ }^{1,2}$, MClinPharm, BPharm(Hons), Rizah Mazzuin Razali ${ }^{3}$, MMEd, MBBS, Shubashini Gnanasan ${ }^{2}$, PhD, MClinPharm, Rosmaliah $\underline{\text { Alias }}^{4}$, MClinPharm, BPharm(Hons), Mahmathi Karuppannan ${ }^{2}$, PhD, MClinPharm

\section{INTRODUCTION}

One of the methods to prevent medication errors is conducting a medication reconciliation process to detect and correct any medication discrepancies. A medication discrepancy occurs when there is a difference between the drugs in the treatment regimen (in term of dose, route and frequency of administration) during transition from one healthcare personnel or health centre to another. ${ }^{(1)}$ A study conducted in Malaysia showed that $49 \%$ of adults discharged from an internal ward had at least one medication discrepancy, ${ }^{(2)}$ while $23.3 \%$ of the elderly discharged from a tertiary care hospital in Singapore had a medication discrepancy upon discharge. ${ }^{(3)}$

Medication discrepancies may cause medication errors and potential harm to patients if left uncorrected. They were identified as contributors to the occurrence of adverse drug events in hospitalised and discharged patients. ${ }^{(4)}$ According to Feleke et al, the number of discharge medications, length of hospitalisation and age did not have a statistically significant association with the high number of medication discrepancies. ${ }^{(5)}$ However, other studies found that the number of medications prescribed upon discharge is the major contributor to medication discrepancies..$^{(2,6)}$ It is thus important to identify the factors that may increase the chance of medication discrepancies occurring, especially in the elderly, as they are prone to having multiple comorbidities and several clinic appointments.

There are two types of discrepancies: intentional and unintentional. Intentional discrepancies happen due to underlying comorbidities or a change in the drug metabolism of the patient. ${ }^{(7)}$ In contrast, unintentional discrepancies are made accidentally by the prescriber and/or due to lack of documentation in clinical records. ${ }^{(6)}$ In identifying these discrepancies, a medication reconciliation process can be implemented, in which healthcare personnel will record the previous medication history from either the patient or the caregiver to ensure that they have the most updated medication list. Prior to the discharge or transfer of the patient, healthcare personnel will compare the medication list obtained during admission with the medications prescribed. Discrepancies can be minimised in several ways. A standardised formal medication reconciliation programme at every point of care should be able to detect and reduce the number of medication discrepancies, as suggested by Salameh et al. ${ }^{(8)}$
Currently, medication reconciliation is not a common practice among healthcare professionals in Malaysia. Involvement of pharmacists and other healthcare professionals is important to reduce the number of discrepancies. Thus, the present study was conducted to determine the prevalence and risk factors of medication discrepancies at discharge among patients who were admitted to the geriatric ward in a tertiary hospital.

\section{METHODS}

We carried out a retrospective study from October to December 2018 at a tertiary care hospital with a dedicated geriatric unit. Patients who were discharged between January and April 2018, and were aged 60 years and above with at least one prescribed medication upon discharge were included in this study. The exclusion criteria included patients who passed away before hospital discharge or were discharged with only a verbal prescription.

A previous study in Malaysia reported a prevalence of medication discrepancies of $49 \%$, which we used in the calculation of our sample size using the Hulley formula. ${ }^{(9)}$ Approximately 68 patients were required for this study. This number was inflated by $10 \%$ to cover for missing information during data collection, thus giving a final sample size of 75 patients.

A pre-prepared data collection form was used. Patients' medical records were reviewed to obtain information regarding age, gender, number of comorbidities, length of hospitalisation, number of medications on admission and upon discharge, primary diagnosis, whether the patient was discharged on a weekday or weekend, and their Modified Barthel Index and Mini-Mental State Examination scores.

The medications list on admission was obtained from the medication history assessment form documented by the ward pharmacist. In this study, only regular medications that were taken daily by the patient for the past two months prior to admission were added to the list; the study panel decided not to include medications taken on an as-needed basis. A comparison of the medication list, in-patient medication chart and medications prescribed upon discharge was performed to identify discrepancies, which were subsequently evaluated by the pharmacist (study researcher) and verified with an experienced

${ }^{1}$ Pharmacy Department, Hospital Queen Elizabeth II, Ministry of Health Malaysia, Kota Kinabalu, Sabah, ${ }^{2}$ Faculty of Pharmacy, Puncak Alam Campus, Universiti Teknologi MARA, Selangor, ${ }^{3}$ Geriatric Unit, Department of Medicine, ${ }^{4}$ Pharmacy Department, Hospital Kuala Lumpur, Ministry of Health Malaysia, Kuala Lumpur, Malaysia

Correspondence: Ms Izzati binti Abdul Halim Zaki, Lecturer, Faculty of Pharmacy, Universiti Teknologi MARA, Puncak Alam Campus, 42300 Bandar Puncak Alam, Selangor, Malaysia.izzatizaki@gmail.com 
geriatric pharmacist to determine whether these discrepancies were documented in the patients' medical files. All documented discrepancies were then reviewed and discussed with a geriatrician, and a clinical judgement was made to determine if there was a justified cause for intentional discrepancies. Otherwise, discrepancies were reported as unintentional. If there was a disagreement, the pharmacist and geriatrician would re-evaluate and discuss the discrepancies until consensus was reached.

Categorical data was reported as counts and percentages. Continuous data was summarised in means and standard deviations where appropriate. All data entry and analyses were carried out using IBM SPSS Statistics version 23.0 (IBM Corp, Armonk, NY, USA), and results were considered statistically significant when $p<0.1$. In identifying the risk factors of medication discrepancies, multivariable linear regression analysis was conducted. The dependent variable was the total number of medication discrepancies, regressed against potential risk factors variables.

Ethics approval was given by the Medical Research and Ethics Committee, Ministry of Health Malaysia (NMRR-18-2538-43585) and Research Ethics Committee of Universiti Teknologi MARA, Malaysia.

\section{RESULTS}

During the study period, 84 cases with complete discharge summaries and medication history assessment forms were screened from 166 patients who were discharged from the geriatric ward of Hospital Kuala Lumpur from January to April 2018. However, only 74 samples with complete case notes were eligible for inclusion.

Most of the patients included in this study were aged $75-84$ years $(n=34,45.9 \%)$ with a mean age of $77.8 \pm 7.2$ years. More than $50 \%$ of the patients included in this study were female $(n=50,67.6 \%)$. The mean duration of hospitalisation was 14.62 \pm 10.09 days, with the majority of the patients being discharged from the ward between Monday and Friday $(81.1 \%, n=60)$. The mean number of comorbidities on admission was $3.41 \pm 1.74$. Out of the 74 patients, 59 (79.7\%) had hypertension diagnosed prior to admission, while another 44 (59.5\%) were previously diagnosed with diabetes mellitus. The number of patients taking 6-10 medications prior to admission was found to be 34 (45.9\%). $54.1 \%$ of the study population were prescribed $6-10$ medications upon discharge. $9(12.2 \%)$ patients were discharged with ten medications. The mean number of medications prescribed at discharge was $7.77 \pm 2.99$.

The medications list upon admission was obtained from various sources, including interviews with caregivers, clinic prescriptions and discharge summaries. On reconciliation of the 74 patients' medications, 1,056 medications were screened for discrepancies. 689 (65.2\%) discrepancies were detected, with a mean number of $9.31 \pm 4.02$ discrepancies detected per patient. The highest number of medication discrepancies was 17 . Two patients had only one discrepancy, which was the lowest number of discrepancies detected in this study.
Table I. Simple linear regression analysis to identify risk factors for medication discrepancies.

\begin{tabular}{|lll|}
\hline Independent variable & $\boldsymbol{b}(\mathbf{9 5} \% \mathbf{C l})$ & $\mathbf{p}$-value* \\
\hline Age $(\mathrm{yr})$ & $0.02(-0.11,0.15)$ & 0.785 \\
\hline Gender & $2.13(-0.19,4.06)$ & 0.030 \\
\hline Length of stay (day) & $0.02(-0.07,0.12)$ & 0.613 \\
\hline Days of discharge & $1.38(-0.99,3.75)$ & 0.250 \\
\hline No. of comorbidities & $0.47(-0.07,1.00)$ & 0.085 \\
\hline No. of medications on admission & $0.53(-0.31,0.75)$ & $<0.001$ \\
\hline No. of medications on discharge & $0.69(-0.42,0.96)$ & $<0.001$ \\
\hline MBI score & $0.00(-0.03,0.04)$ & 0.817 \\
\hline MMSE score & $0.01(-0.19,0.21)$ & 0.911 \\
\hline
\end{tabular}

${ }^{*} \mathrm{p}<0.10$ is statistically significant. $b$ : crude regression coefficient; $\mathrm{Cl}$ : confidence interval; MBI: Modified Barthel Index; MMSE: Mini-Mental State Examination

Medication discrepancies were further classified into addition, omission, dose changes and frequency changes. Of the 689 discrepancies detected, 533 (77.4\%) were intentional discrepancies and the remaining 156 (22.6\%) were unintentional discrepancies. The most common intentional discrepancy was additions $(57.8 \%, \mathrm{n}=308)$ and the least common intentional discrepancy was frequency changes $(7.5 \%, \mathrm{n}=40)$. Omissions were the most common unintentional discrepancy $(64.7 \%$, $\mathrm{n}=101)$ followed by additions $(23.7 \%, \mathrm{n}=37)$ and dose changes $(9.0 \%, \mathrm{n}=14)$.

Simple linear regression analysis was conducted to predict the factors for a multivariable regression analysis to identify risk factors associated with the number of medication discrepancies. Nine predictors were included in the model (Table I). In this study, a $90 \%$ confidence level was utilised due to the small sample size. Hence, variables with $\mathrm{p}<0.1$ were considered as significant. Findings from the simple linear regression analysis demonstrated that gender, number of comorbidities, number of medications upon admission and number of medications upon discharge had a significant association with the dependent variable.

Findings from the multiple linear regression analysis demonstrated that the number of medications upon admission and upon discharge were significantly associated with the dependent variable (Table II). There was a positive association between an increase in the number of medications on admission and the total number of medication discrepancies.

\section{DISCUSSION}

The number of medication discrepancies reported in our study $(65.2 \%)$ is higher than the $49 \%$ that was reported among adult patients discharged from another medical ward in Malaysia. ${ }^{(2)}$ However, our finding is consistent with previous studies conducted in other countries, which reported the prevalence of medication discrepancies to be between $20.0 \%$ and $87.2 \% .^{(5,7,10-13)}$ The differences in prevalence could be due to varying definitions and interpretations used by investigators on medication discrepancies. The various methodological approaches and taxonomies used across studies could also explain the differing prevalence of discrepancies reported. ${ }^{(7)}$ 
Table II. Multivariable linear regression analysis of the factors associated with total number of medication discrepancies among the study population $(n=74)$.

\begin{tabular}{|c|c|c|c|c|c|}
\hline \multirow[t]{2}{*}{ Independent variable } & \multicolumn{2}{|c|}{ Simple linear regression } & \multicolumn{3}{|c|}{ Multivariable linear regression } \\
\hline & $b(95 \% \mathrm{Cl})$ & p-value & Adj $b(95 \% \mathrm{Cl})$ & t-stat & p-value \\
\hline Gender & $2.13(0.19,4.06)$ & 0.030 & - & - & - \\
\hline No. of comorbidities & $0.47(-0.07,1.00)$ & 0.085 & - & - & - \\
\hline No. of medications on admission & $0.53(0.31,0.75)$ & $<0.001$ & $0.37(0.15,0.59)$ & 3.33 & $<0.001$ \\
\hline No. of medications on discharge & $0.69(0.42,0.96)$ & $<0.001$ & $0.51(0.24,0.79)$ & 3.73 & $<0.001$ \\
\hline
\end{tabular}

$\operatorname{Adj} b$ : adjusted regression coefficient; b: crude regression coefficient; $\mathrm{Cl}$ : confidence interval

The mean number of medication discrepancies per patient reported in our study was 9.31, which is higher than in studies conducted in Saudi Arabia and North Norway that reported 4.7 and 5.6 medication discrepancies per patient, respectively. ${ }^{(6,14)}$ The higher number of discrepancies in our study could be due to the different setting and study population. The lower number of medication discrepancies in other settings may be influenced by the availability of an electronic records system, which was not available in this study. Having such a system can help to ease the documentation process during physicians' rounds and indirectly reduce the number of medication discrepancies in the ward. Hospital Kuala Lumpur is a tertiary referral hospital that uses a manual medical recording system. All interventions, findings and plans need to be written by the healthcare professionals in a dedicated patient's file. The file is sent to the record office once the patient is discharged from the ward, and should be available to be traced when the patient returns for a follow-up or re-admission.

Further classification of the discrepancies during the reconciliation process found that $77.4 \%$ were intentional discrepancies and $22.6 \%$ were unintentional discrepancies. This is similar to a study conducted among the elderly in Singapore by Akram et al, who found that $23.3 \%$ of patients had at least one unintentional medication discrepancy on discharge. ${ }^{(3)}$ However, this number is lower compared to the number of unintentional medication discrepancies reported among adult patients discharged from a tertiary hospital in Sabah (40\%) and in patients who were admitted to a hospital in Jordan (35\%). ${ }^{(2,8)}$

The lower percentage of unintentional medication discrepancies reported in this study could be due to the characteristics of the included study population. All eligible patients were recruited, including patients without any medication prior to admission. This may have indirectly reduced the likelihood of medication discrepancies upon discharge. In a similar study conducted in Singapore, the practice of daily clinical rounds by a geriatrician may have resulted in a lower number of unintentional medication discrepancies among elderly patients. ${ }^{(3)}$

Identifying risk factors for medication discrepancies provides healthcare professionals with critical information to ensure patient safety and prevent medication errors. Two predictors were found to be risk factors for medication discrepancy upon discharge among elderly patients in our study: number of medications on admission and number of medications upon discharge. Having a greater number of medications on admission was positively related to the number of medication discrepancies upon discharge from the ward, a finding that is similar to several earlier studies..$^{(1,12,15)}$ In addition, the risk of medication discrepancies was also predicted through the number of medications prescribed prior to discharge. A comparable finding was reported by another study in Malaysia, which found that the number of medication discrepancies was 1.2 times higher with every additional medication prescribed prior to discharge. ${ }^{(2)}$ The simple conclusion we can draw from these findings is that if more medications are prescribed (whether on admission or upon discharge), there are likely to be more medication discrepancies.

Findings from this study should be interpreted in light of several limitations. First, as this study was conducted retrospectively, investigators were unable to discuss with physicians nor talk to patients and caregivers when reviewing the two medication lists. Second, this study was conducted in a single centre with a small sample size, resulting in limited generalisability to older adult populations in general medical wards. Third, there is currently no gold standard in identifying medications taken by a patient at home. It was assumed that medications taken by the patient at home prior to the hospital admission were correct. However, this limitation was mitigated by using numerous sources of information, including the medication container, the patient and his/her family members, discharge summaries, electronic medical records, and pharmacy records. Lastly, the classification of discrepancies as intentional and unintentional relied on the subjective agreement of the panel, which may be a source of bias.

Despite the limitations highlighted, preventive measures should be taken at all stages of care to prevent discrepancies. A thorough medication history assessment by healthcare professionals on admission is an important part of the reconciliation process. It is important to have proper documentation of any changes made to the patients' medication list throughout the hospital stay and upon discharge to prevent medication errors. Any changes made to the treatment regimen during admission should be recorded in a detailed manner for the next healthcare professionals to ensure continuity of care.

Medication discrepancies are common among discharged elderly patients. The results from this study highlight the importance of a comprehensive medication history assessment on admission and medication reconciliation process prior to discharge. All healthcare professionals should be able to conduct the medication reconciliation process to detect discrepancies during transitions of care. 


\section{REFERENCES}

1. Okerosi EK, Okalebo FA, Opanga SA, Guantai AN. Prevalence and risk factors for medication discrepancies on admission of elderly diabetics at Kenyatta National Hospital, Kenya. African J Pharmacol Ther 2017; 6:54-63.

2. Law BK, Chong CP. Prevalence and predictors of medication discrepancies upon discharge among adult patients: a prospective study from Malaysia. J Asian Assoc Sch Pharm 2017; 6:1-9.

3. Akram F, Huggan PJ, Lim V, et al. Medication discrepancies and associated risk factors identified among elderly patients discharged from a tertiary hospital in Singapore. Singapore Med J 2015; 56:379-84.

4. Pippins JR, Gandhi TK, Hamann C, et al. Classifying and predicting errors of inpatient medication reconciliation. J Gen Intern Med 2008; 23:1414-22.

5. Feleke M, Berha AB, Shibeshi W. Reconciliation of medication discrepancies at hospital discharge for inpatients in medical ward of St. Paul's Hospital Millennium Medical College, Addis Ababa, Ethiopia. Ann Clin Lab Res 2015; 3:4.

6. Al-Rashoud I, Al-Ammari M, Al-Jadhey H, et al. Medication discrepancies identified during medication reconciliation among medical patients at a tertiary care hospital. Saudi Pharm J 2017; 25:1082-5.

7. Michaelsen M, McCague P, Bradley CP, Sahm LJ. Medication reconciliation at discharge from hospital: a systematic review of the quantitative literature. Pharmacy (Basel) 2015; 3:53-71.
8. Salameh L, Abu Farha R, Basheti I. Identification of medication discrepancies during hospital admission in Jordan: prevalence and risk factors. Saudi Pharm J 2017; 26:125-32

9. Hulley SB, Cummings SR, Browner WS, Grady DG, Newman TB. Designing clinical research. $4^{\text {th }}$ ed. Philadelphia: Lippincott Williams \& Wilkins, 2013.

10. Forjuoh SN, Reis MD, Couchman GR, et al. Physician response to written feedback on a medication discrepancy found with their elderly ambulatory patients. J Am Geriatr Soc 2005; 53:2173-7.

11. Hafferty JD, Campbell AI, Navrady LB, et al. Self-reported medication use validated through record linkage to national prescribing data. J Clin Epidemiol 2018; 94:132-42.

12. Herrero-Herrero Jl, García-Aparicio J. Medication discrepancies at discharge from an internal medicine service. Eur J Intern Med 2011; 22:43-8.

13. Knez L, Suskovic S, Rezonja R, Laaksonen R, Mrhar A. The need for medication reconciliation: a cross-sectional observational study in adult patients. Respir Med 2011; 105 Suppl 1:S60-6

14. Holmstad B, Elenjord R, Svendsen K, Garcia B. Medication reconciliation in North Norway; does a written procedure reduce medication discrepancies in medication charts in hospital? Conference paper. Int J Clin Pharm 2015; 37:23-4.

15. Poornima P, Reshma P, Ramakrishnan TV, et al. Medication reconciliation and medication error prevention in an emergency department of a tertiary care hospital. J Young Pharm 2015; 7:241-9 